\title{
THE
}

\section{Dynamics of quantum spin chains and multi-fermion excitation continua}

Oleg Derzhko

Taras Krokhmalskii

Joachim Stolze

Gerhard Müller

University of Rhode Island, gmuller@uri.edu

Follow this and additional works at: https://digitalcommons.uri.edu/phys_facpubs

Terms of Use

All rights reserved under copyright.

\section{Citation/Publisher Attribution}

Derzhko, O., Krokhmalskii, T., Stolze, J., \& Müller, G. (2006). Dynamics of quantum spin chains and multifermion excitations. Physica B: Condensed Matter. 378-380, 445-446. doi: 10.1016/j.physb.2006.01.192 Available at: http://dx.doi.org/10.1016/j.physb.2006.01.192 


\title{
Dynamics of quantum spin chains and multi-fermion excitation continua
}

\author{
O. Derzhko a,*, T. Krokhmalskii a ${ }^{\text {a }}$ J. Stolze ${ }^{\mathrm{b}}$, G. Müller $^{\mathrm{c}}$ \\ ${ }^{\mathrm{a}}$ Institute for Condensed Matter Physics NASU, 1 Svientsitskii Str., L'viv-11, 79011, Ukraine \\ ${ }^{\mathrm{b}}$ Institut für Physik, Universität Dortmund, 44221 Dortmund, Germany \\ ${ }^{\mathrm{c}}$ Department of Physics, University of Rhode Island, Kingston, Rhode Island 02881-0817, USA
}

\begin{abstract}
We use the Jordan-Wigner representation to study dynamic quantities for the spin- $\frac{1}{2} X X$ chain in a transverse magnetic field. We discuss in some detail the properties of the four-fermion excitation continuum which is probed by the dynamic trimer structure factor.
\end{abstract}

Key words: spin- $\frac{1}{2} X X$ chain, dynamic quantities, multi-fermion excitations PACS: $75.10 . \mathrm{Jm}$

Recently the subject of multi-magnon excitations of quasi-one-dimensional quantum spin systems has attracted considerable interest. With high-resolution inelastic neutron scattering experiments one may expect to examine not only the bound two-magnon states but also the continua of multi-magnon states. Some properties of multi-magnon continua were examined in [1]. More recently, we have noted that the spin- $\frac{1}{2}$ transverse $X X$ chain, which can be mapped via the Jordan-Wigner transformation onto noninteracting spinless fermions, is a model whose dynamics is governed by continua of multi-fermion excitations. In particular, the dynamic trimer structure factor involves two-fermion and four-fermion excitations [2]. In the present report we compare and contrast the general and specific properties of the four-fermion excitation continuum, which contributes to the dynamics of trimer fluctuations.

To be specific, we consider the spin- $\frac{1}{2}$ transverse $X X$ chain with the Hamiltonian

\footnotetext{
* Corresponding author. Tel: +38 0322 761978, fax: +38 0322 761158

Email address: derzhko@icmp.lviv.ua (O. Derzhko).
}

$$
H=\sum_{n} J\left(s_{n}^{x} s_{n+1}^{x}+s_{n}^{y} s_{n+1}^{y}\right)+\sum_{n} \Omega s_{n}^{z} .
$$

We will set further $J=-1$. The trimer operator is defined as $T_{n}=s_{n}^{x} s_{n+2}^{x}+s_{n}^{y} s_{n+2}^{y}$ and the corresponding dynamic structure factor

$$
S_{T T}(\kappa, \omega)=\sum_{l} \mathrm{e}^{-\mathrm{i} \kappa l} \int_{-\infty}^{\infty} \mathrm{d} t \mathrm{e}^{\mathrm{i} \omega t}\left\langle\Delta T_{n}(t) \Delta T_{n+l}(0)\right\rangle,
$$

$\Delta T_{n}(t)=T_{n}(t)-\langle T\rangle$ can be written as a sum of the two-fermion contribution $S_{T T}^{(2)}(\kappa, \omega)$ and the fourfermion contribution $S_{T T}^{(4)}(\kappa, \omega)$ with

$$
\begin{array}{r}
S_{T T}^{(2)}(\kappa, \omega)=\int \mathrm{d} \kappa_{1} \mathrm{~d} \kappa_{2} C^{(2)}\left(\kappa_{1}, \kappa_{2}\right) n_{\kappa_{1}}\left(1-n_{\kappa_{2}}\right) \\
\cdot \delta\left(\omega+\Lambda_{\kappa_{1}}-\Lambda_{\kappa_{2}}\right) \delta_{\kappa+\kappa_{1}-\kappa_{2}, 0}, \\
S_{T T}^{(4)}(\kappa, \omega)=\frac{1}{4 \pi^{2}} \int \mathrm{d} \kappa_{1} \ldots \mathrm{d} \kappa_{4} C^{(4)}\left(\kappa_{1}, \ldots, \kappa_{4}\right) \\
\cdot n_{\kappa_{1}} n_{\kappa_{2}}\left(1-n_{\kappa_{3}}\right)\left(1-n_{\kappa_{4}}\right) \\
\cdot \delta\left(\omega+\Lambda_{\kappa_{1}}+\Lambda_{\kappa_{2}}-\Lambda_{\kappa_{3}}-\Lambda_{\kappa_{4}}\right) \delta_{\kappa+\kappa_{1}+\kappa_{2}-\kappa_{3}-\kappa_{4}, 0} .
\end{array}
$$

Here $C^{(2)}\left(\kappa_{1}, \kappa_{2}\right), C^{(4)}\left(\kappa_{1}, \ldots, \kappa_{4}\right) \geq 0$ are certain functions the explicit expressions for which are given in $[2], n_{\kappa}=\left(1+\exp \left(\beta \Lambda_{\kappa}\right)\right)^{-1}$ is the Fermi function, $\Lambda_{\kappa}=$ 
$\Omega+J \cos \kappa$, and $-\pi \leq \kappa<\pi$ is the quasi-momentum which parameterizes the Jordan-Wigner fermions. It is easy to note that Eq. (3) coincides with the dynamic $z z$ structure factor if $C^{(2)}\left(\kappa_{1}, \kappa_{2}\right)=1$ or with the dynamic dimer structure factor if $C^{(2)}\left(\kappa_{1}, \kappa_{2}\right)=$ $\cos ^{2} \frac{\kappa_{1}+\kappa_{2}}{2}$ (see [2]). These dynamic quantities are governed exclusively by the two-fermion (one particle and one hole) excitations. The properties of the twofermion excitation continuum were examined in [3,4].

In contrast, Eq. (4) is governed exclusively by the four-fermion (two particles and two holes) excitation continuum the properties of which are described concisely below. The specific features of the four-fermion contribution to $S_{T T}(\kappa, \omega)$ (2) are controlled by the function $C^{(4)}\left(\kappa_{1}, \ldots, \kappa_{4}\right)$. To display the generic behavior of a four-fermion dynamic quantity we also consider Eq. (4) with $C^{(4)}\left(\kappa_{1}, \ldots, \kappa_{4}\right)=1$ (compare Figs. 1 and 2).

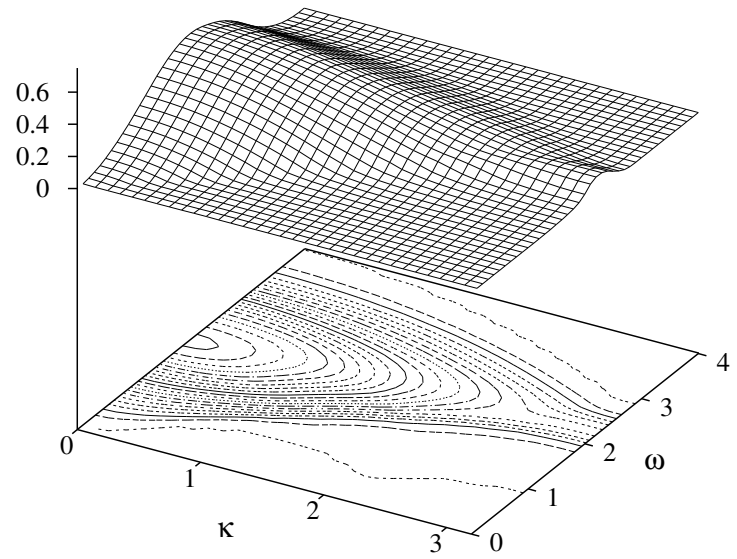

Fig. 1. $S_{T T}^{(4)}(\kappa, \omega)$ (4) for the chain (1) with $J=-1, \Omega=0.25$ at zero temperature $(\beta \rightarrow \infty)$.

The four-fermion dynamic quantity can have nonzero values only in a restricted region of the $\kappa-\omega$ plane. At nonzero temperatures one immediately finds the upper boundary of the four-fermion excitation continuum, $4|J| \cos \frac{\kappa}{4}$. At zero temperature the Fermi functions in (4) come into play and both the upper and the lower boundaries of the four-fermion excitation continuum become complicated $\Omega$-dependent functions of $\kappa$. For $\Omega=0.25$ the upper boundary remains $4|J| \cos \frac{\kappa}{4}$, whereas the lower boundary assumes the following values as $\kappa$ increases from 0 to $\pi: \omega_{l}^{(1)}=$ $2|J| \sin \frac{\kappa}{2} \sin \left(\alpha-\frac{\kappa}{2}\right), \omega_{l}^{(2)}=4|J| \cos \frac{\kappa}{4} \cos \left(\alpha+\frac{\kappa}{4}\right)$, $\omega_{l}^{(3)}=-2|J| \sin \left(\alpha+\frac{\kappa}{2}\right) \sin \left(2 \alpha+\frac{\kappa}{2}\right), \omega_{l}^{(1)}, \omega_{l}^{(4)}=$ $-2|J| \sin \left(\alpha-\frac{\kappa}{2}\right) \sin \left(2 \alpha-\frac{\kappa}{2}\right)$ with $\cos \alpha=\frac{\Omega}{|J|}$. To find these boundaries we (numerically) seek for the extrema of $\cos \kappa_{1}+\cos \kappa_{2}-\cos \kappa_{3}-\cos \kappa_{4}$ with the restrictions imposed by the Fermi functions (see (4))

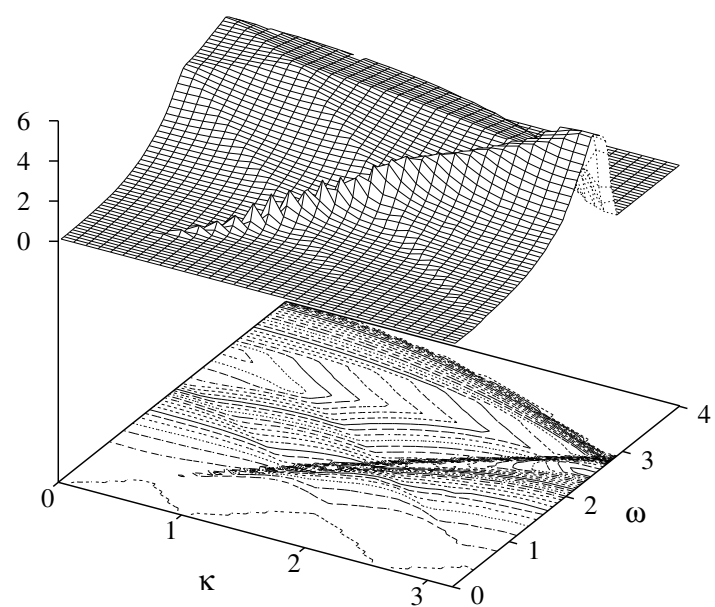

Fig. 2. The same as in Fig. 1 but with $C^{(4)}\left(\kappa_{1}, \ldots, \kappa_{4}\right)=1$.

for $0 \leq \kappa \leq \pi$ and determine the values of $\kappa_{1}, \ldots, \kappa_{4}$ at which such extrema occur. We find simple relations between the quantities $\kappa_{1}, \ldots, \kappa_{4}$ and $\kappa, \alpha$ obtaining as a result the upper and the lower boundaries of the four-fermion excitation continuum.

The four-fermion dynamic quantities may exhibit Van Hove cusp singularities akin to the three dimensional density of states. These singularities occur along the lines $2|J| \sin \frac{\kappa}{2}, 4|J| \sin \frac{\kappa}{4}$, and $4|J| \cos \frac{\kappa}{4}$.

Comparing Figs. 1 and 2 we see how some characteristic features of the four-fermion excitation continuum are smeared out owing to $C^{(4)}\left(\kappa_{1}, \ldots, \kappa_{4}\right) \neq 1$.

Finally, we note that spin- $\frac{1}{2} X X$ chains are realized in some quasi-one-dimensional magnetic insulators (e.g. such as $\mathrm{Cs}_{2} \mathrm{CoCl}_{4}$ [5]). The dynamic dimer structure factor is relevant to phonon-assisted optical adsorption $[6,7]$; the direct experimental relevance of the dynamic trimer structure factor is less evident. However, our results may be important from the theoretical point of view since the four-fermion dynamic trimer structure factor is a quantity of intermediate complexity between the two-fermion dynamic $z z$ structure factor and the multi-fermion dynamic $x x(y y)$ structure factor.

This study was supported by the STCU under the project \#1673.

References

[1] T. Barnes, Phys. Rev. B 67 (2003) 024412.

[2] O. Derzhko et al., Phys. Rev. B 71 (2005) 104432.

[3] G. Müller et al., Phys. Rev. B 24 (1981) 1429.

[4] J. H. Taylor and G. Müller, Physica A 130 (1985) 1.

[5] M. Kenzelmann et al., Phys. Rev. B 65 (2002) 144432. 
[6] H. Suzuura et al., Phys. Rev. Lett. 76 (1996) 2579.

[7] J. Lorenzana and R. Eder, Phys. Rev. B 55 (1997) R3358. 